\title{
El uso del archivo desde la perspectiva de los usuarios El caso del Archivo Nacional Histórico de Chile
}

\author{
Gabriela Andaur Gómez \\ Universidad Alberto Hurtado, Facultad de Economía y Negocios. Departamento de Gestión y Negocios. Carrera Gestión de \\ Información, Bibliotecología y Archivística. Santiago, Chile / gandaur@uahurtado.cl / https://orcid.org/oooo-ooo3-2438-1074
}

\begin{abstract}
Resumen
En este artículo se presentan los resultados de un estudio de usuarios realizado durante el año 2016 en el Archivo Nacional Histórico de Chile, que tuvo como objetivo explorar la experiencia de los usuarios en el acceso a los documentos preservados en dicho archivo, mediante la caracterización y comprensión de su experiencia en el uso de los servicios de consulta presencial de documentos, la identificación de sus necesidades y de los elementos más significativos para su satisfacción, utilizando como técnicas el grupo focal y la encuesta. Se presentan los resultados en los ámbitos de experiencia, necesidades y satisfacción e identifican los principales ámbitos que inciden en la experiencia de los usuarios: infraestructura y equipamiento; condiciones del servicio y procedimientos; instrumentos descriptivos y herramientas de búsque$\mathrm{da}, \mathrm{y}$ rol de funcionarios. Por otra parte, se presentan reflexiones metodológicas sobre el uso de técnicas mixtas de investigación de forma complementaria, en pos de una mayor representación y comprensión de la diversidad de los usuarios y sus experiencias en archivos. Finalmente, se presentan algunas recomendaciones para el estudio de los usuarios del Archivo Nacional Histórico y se sugieren líneas de investigación que puedan aportar al conocimiento de los usuarios en este y otros archivos.
\end{abstract}

\footnotetext{
Abstract

The use of archives from the users' perspective: The case of the Archivo Nacional Histórico de Chile. This article presents the findings of a study conducted in 2016 at the Archivo Nacional Histórico de Chile, aimed at exploring the experience of users in accessing records preserved by the institution. The study sought to characterise and understand the experiences of in situ onsite consultations of documents and to identify their needs and the most significant elements for their satisfaction, using focus groups and surveys as research techniques. The paper presents findings in the areas of user experience, needs, and satisfaction, and identifies the most relevant aspects in the overall experience of participating users: infrastructure and equipment; service conditions and procedures; finding aids systems, and role of archival reference personnel. The article also reflects the use of mixed research techniques
}

\section{Palabras clave}

Estudio de usuarios Archivos Chile

\section{Keywords}

User study Archives Chile 
to better represent and understand the diversity of archives users and their experiences. Finally, it presents recommendations for the study of users of the Archivo Nacional Histórico de Chile and future research suggestions that may contribute to the knowledge of users of archives in general.

Artículo recibido: 7-12-2017. Aceptado: 30-03-2018

1. El presente artículo se enmarca en la ejecución del proyecto "Percepciones, nivel de satisfacción y necesidades de usuarios de archivos públicos. Estudio de caso en el Archivo Nacional Histórico de Santiago de Chile", financiado por el Fondo de Fomento a la Investigación de la Universidad Alberto Hurtado (Chile). Los resultados fueron parcialmente presentados en el XII Congreso de Archivología del Mercosur en septiembre de 2016, gracias al financiamiento otorgado por el Departamento de Gestión y Negocios de la Facultad de Economía y Negocios de la misma universidad.

2. Dentro de este ámbito también es posible encontrar un mayor desarrollo teórico de los estudios de usuario. Ejemplo de ello es el trabajo de Juan José Calva González (2004), que apunta a un abordaje que considere de forma integral tres ámbitos interconectados que generalmente se estudian de forma separada: el surgimiento de las necesidades de información, su manifestación (es decir, el comportamiento en la búsqueda de información) y, finalmente, la satisfacción de los usuarios

\section{Introducción ${ }^{1}$}

El acceso a la información está en el centro de la labor de los archivos. La literatura archivística -incluyendo manuales y artículos científicos- repite constantemente esta idea, enfatizando que la misión de preservar documentos solo cobra sentido en la medida en que estos son puestos a disposición de una determinada comunidad.

En este contexto, puede entenderse la necesidad de comprender la forma en que cada comunidad accede a la documentación, así como a otros servicios que puedan ser implementados por el archivo en el cumplimiento de su misión. Para ello existen los estudios de usuarios, entendidos como "el conjunto de estudios que tratan de analizar cualitativa y cuantitativamente los hábitos de información de los usuarios, mediante la aplicación de distintos métodos" (Sanz Casado, 1994: 31) lo que permite, además, "analizar diferentes aspectos y características de la relación que se establece entre la información y el usuario" (Martín Moreno, 2007: 133)

En la literatura generada dentro del ámbito iberoamericano se ha valorado este tipo de estudios como una herramienta para el conocimiento de los usuarios, la evaluación de los servicios y la planificación e implementación de mejoras en unidades de información de distinto tipo. Así, sabemos que este tipo de estudios permite conocer y analizar el perfil de los usuarios, sus hábitos y necesidades de información, la estructura y dinámica de los colectivos de investigación, y las interacciones de los usuarios con el archivo. En segundo lugar, los estudios de usuarios hacen posible la evaluación de la calidad y eficacia de servicios y productos, y del uso de los recursos empleados. Finalmente, esta información resulta indispensable para identificar las áreas de mejora e introducir las reformas que sean necesarias para el ofrecimiento de servicios de calidad. En síntesis, "solamente por medio de ellos, se determina de forma científica el consumo, las necesidades y los hábitos de información archivística de los clientes, lo que contribuye a una acertada toma de decisiones y a evaluar la efectividad del sistema de información" (Campos Ramírez, 2009).

No obstante, aunque ha sido observada la realización de estudios de usuarios en archivos desde la década de los 90 (Allendez Sullivan y de la Puente, 2013:4), el volumen de publicaciones en este ámbito sigue siendo moderado, con mayor presencia en el ámbito de la bibliotecología. ${ }^{2}$ En cuanto al ámbito archivístico, existe un número acotado de publicaciones en países de habla hispana, que generalmente se dividen entre las que profundizan en la descripción de los estudios de usuarios desde un punto de vista teórico y metodológico, y aquellas que presentan experiencias concretas en el estudio de los usuarios de uno o más archivos.

Dentro del abordaje teórico de los estudios de usuarios en el ámbito de los archivos, ha emergido como aspecto relevante el de la tipología. Así, la diversidad de usuarios ha dado paso a la elaboración de tipologías basadas en distintos criterios. Mientras algunas tipologías se basan en el tipo de vínculo del usuario con el archivo, distinguiendo entre usuarios internos y externos, otras se centran en el uso efectivo del 
archivo y su frecuencia, separando a usuarios reales de los potenciales (Allendez Sullivan y de la Puente, 2013). También se han establecido diferencias basadas en el ámbito de ocupación, las motivaciones y el nivel de dominio sobre el archivo, como la frecuentemente citada tipología de Sanz Casado (1994) o la de Jaén García (2006). Por último, autores como Lara Navarra y Ruiz Rodríguez (1999) han establecido distinciones según la capacidad y las condiciones que posibilitan o limitan el uso de los archivos por parte de ciertos sectores de la sociedad. ${ }^{3}$

En cuanto a los métodos, la literatura ha hecho alusión de forma recurrente a las características del método directo e indirecto, siendo el primero aquel que utiliza instrumentos y técnicas creadas especialmente para "que sea el propio usuario quien defina sus hábitos, necesidades o el uso que hace de la información que necesita" (Sanz Casado, 1994: 91), tales como cuestionarios, entrevistas o grupos focales. El segundo, en cambio, se basa en "la recopilación de los datos o información proporcionada por parte de los clientes, sin tener que consultarlos a ellos" (Jaén García, 2006: 5), presente en fuentes tales como el registro de usuarios, de préstamos o de reprografía.

Finalmente, en cuanto a aquellos trabajos que presentan los resultados de estudios de caso en archivos específicos, se puede mencionar que han estado orientados principalmente a caracterizar a los usuarios, identificar tipologías y necesidades de información y evaluar la satisfacción de usuarios. Algunos estudios han utilizado el método indirecto y el análisis de resultados bajo un enfoque cuantitativo, tales como el de Rubio Hernández (2004) en el Archivo Municipal de Logroño y el de Santaella (2005) en el Archivo Histórico Provincial, el del Ayuntamiento y el de Diputación Provincial de Jaén. También se han desarrollado estudios que han combinado el uso del método indirecto y directo, bajo un enfoque mixto (cuantitativo y cualitativo), tales como el completo estudio de Jaén García (2013) en el Archivo Nacional de Costa Rica y el de Acosta Sandoval; Íncer Solís y Mena Aguilar (2006) en los archivos de las municipalidades de San José y Escazú, también en Costa Rica. No obstante, como ya se ha manifestado, el número de estudios de usuarios realizados en el ámbito de los archivos es insuficiente. El conocimiento de los usuarios, sus necesidades de información y su satisfacción no han sido abordados con la frecuencia ni la profundidad necesaria dentro del ámbito archivístico.

Considerando la relevancia de los estudios de usuarios y la utilidad que tienen para la mejora de los servicios ofrecidos en los archivos, este sigue siendo un hecho paradójico y problemático. ¿Cómo puede asegurarse el efectivo acceso a los documentos de archivo y la información que contienen sin conocer las características, necesidades y expectativas de quienes los requieren? ¿Cómo pueden introducirse mejoras relevantes en los servicios archivísticos sin conocer la experiencia de los usuarios ni indagar en la medida en que las condiciones en que se ofrecen dichos servicios aportan a la satisfacción de sus necesidades de información? En un ámbito más amplio, Hernández Salazar (2011) ha enfatizado cómo la satisfacción de estas necesidades no es un aspecto más del trabajo de los profesionales de unidades de información, sino que es el sentido ontológico mismo de la disciplina. En este sentido, es necesario orientar los esfuerzos para hacer posible que los archivos cumplan de forma efectiva el objetivo para el cual fueron creados.

Con el objetivo de avanzar hacia este fin, este trabajo indaga en la experiencia de los usuarios en relación con el acceso a la documentación en archivos, específicamente en el caso del Archivo Nacional Histórico de Chile (ANH). La relevancia del ANH como principal institución responsable de preservar y dar acceso a la documentación histórica producida por organismos públicos en Chile, así como la falta de evidencia publicada sobre la realización de estudios de usuarios tanto en este como en otros archivos en Chile, son hechos relevantes para justificar la pertinencia de este estudio.
3. La tipología de Sanz Casado está compuesta por: el investigador y docente; la industria; el administrador, planificador y político, y el hombre de la calle. La de Jaén García distingue entre: Investigadores profesionales; investigadores aficionados; estudiantes universitarios, y ciudadanos en general. En cuanto al nivel de experiencia y dominio en el uso del archivo, Lara Navarra y Ruiz Rodríguez establecen una tipología que distingue entre: i. Usuario interno, ii. Usuario potencial, iii. Pre-usuario y iv. No-usuario 
4. Se realizó una entrevista de 1 hora de duración al Encargado de Atención de Usuarios del ANH, en septiembre del año 2015. Parte de la información recabada es presentada en el apartado 3.1 de este trabajo.

5. Las sesiones se realizaron los días 15 y 16 de junio del año 2016 y contaron con 8 y 5 participantes, respectivamente.

6. La encuesta se aplicó entre el 23 de noviembre y el 16 de diciembre de 2016. Es importante acotar que la dificultad para determinar el universo de usuarios del ANH, limitó la determinación de una muestra que pudiese ser representativa del conjunto en términos estadísticos.

Esto explica la decisión de no

utilizar los resultados para hacer análisis estadístico pormenorizado.
En términos específicos, esta investigación tiene como objetivos caracterizar las experiencias y las necesidades de los usuarios en relación con los servicios de búsqueda y consulta de documentos e identificar los principales factores que inciden en su satisfacción. Se espera que, mediante el cumplimiento de estos objetivos, la institución cuente con antecedentes que aporten a un mayor conocimiento de los usuarios del $\mathrm{ANH}$, así como a la identificación de ámbitos de mejora en los servicios de consulta de documentos ofrecidos por la institución. Por otra parte, desde la perspectiva metodológica, se espera que este estudio contribuya a la identificación y evaluación de métodos y técnicas idóneas para la comprensión de la experiencia cotidiana y satisfacción de los usuarios que día a día acuden a los archivos para satisfacer sus necesidades de información.

\section{Metodología}

Considerando la falta de experiencias en el ámbito de estudios de usuarios en archivos en Chile y, particularmente, en el Archivo Nacional, el presente estudio es de tipo exploratorio. En cuanto al enfoque, es de tipo mixto, es decir, cuenta con componentes cuantitativos y cualitativos. Al ser un estudio de caso, se estima que los resultados del análisis cualitativo y cuantitativo de la información recabada no podrá extrapolarse a otras instituciones de similar tipo.

De forma previa a esta investigación se realizó un levantamiento preliminar de información, utilizando como técnicas la observación no-participante y la entrevista. Ésta última, de tipo semiestructurada, se realizó a la persona encargada de atención de usuarios en el ANH, y permitió identificar las principales características de la institución y sus servicios, además de recoger algunas impresiones en relación con la atención de usuarios. ${ }^{4}$

Los resultados que aquí se presentan corresponden a la investigación posterior, realizada a lo largo del año 2016. Se utilizaron dos técnicas: el grupo focal y la encuesta. En relación con la primera técnica, se realizaron dos grupos focales en las dependencias del ANH, con una duración que varió entre 60 y 80 minutos. Tras un proceso de invitación presencial realizado en la Sala de Lectura del ANH durante dos semanas, se logró contar con la participación de trece usuarios con distintos niveles de experiencia en el archivo en cuestión. En cada una de las sesiones se utilizó un formulario de consentimiento informado ${ }^{5}$, y se siguió una misma pauta de preguntas organizada en torno a seis temáticas: Experiencias previas en otros archivos; primeras experiencias en el ANH; experiencia cotidiana en el ANH; interacción con funcionarios; áreas de mejora de servicios y, finalmente, percepciones sobre el rol del ANH en la preservación y acceso al patrimonio documental.

Por otra parte, se aplicó una encuesta de tipo autoadministrada en la Sala de Lectura del ANH. La selección de las unidades que componen la muestra fue realizada por conveniencia. Dado que esta es una técnica de muestreo no probabilístico, los resultados no se consideran representativos de la totalidad de la población, sino que se limitan a aquellos usuarios presentes en la Sala en los momentos de aplicación. Se seleccionaron días y horas diferentes dentro de un margen de cuatro semanas, con la intención de captar la mayor diversidad posible de usuarios, lográndose aplicar 93 encuestas en total. ${ }^{6}$ Esta encuesta contó con 19 preguntas -16 de tipo cerrada y 3 abiertas. Las preguntas fueron elaboradas de forma previa al grupo focal y ajustadas de forma posterior a su realización, lo que permitió incorporar temas que emergieron durante cada sesión y que fueron identificados como relevantes por los participantes. La encuesta se dividió en tres secciones destinadas a conocer las características sociodemográficas de los encuestados, el tipo y frecuencia de uso del archivo y su 
nivel de satisfacción en relación con los servicios de consulta presencial en el ANH. Dadas las características de la muestra, no se hizo un análisis estadístico de los resultados, sino que estos fueron analizados en conjunto con los grupos focales, triangulando y complementado la información derivada de cada técnica. Esto permitió observar algunas tendencias generales y divergencias, considerando un grupo de mayor tamaño y diversidad que el que participó en los grupos focales.

\section{Presentación y análisis de resultados}

\subsection{Elementos contextuales: el acceso a los documentos en el ANH}

El Archivo Nacional de Chile (AN) es la institución que preserva y da acceso a la documentación producida por diversos organismos del Estado chileno. Su creación quedó establecida por el Decreto con Fuerza de Ley no. 5200 de 1929, norma vigente hasta el día de hoy, que, tras las últimas modificaciones realizadas en el año 2017, establece la dependencia de esta institución del Servicio Nacional del Patrimonio Cultural. La misión del Archivo Nacional ha quedado establecida en la ley no. 21045 de 2017 (Congreso Nacional de Chile, 2017), que crea el Ministerio de las Culturas, el Arte y el Patrimonio. Según esta norma, el AN

tendrá como misión reunir, organizar, preservar, investigar y difundir el conjunto de documentos, independientemente de su edad, forma o soporte, producidos orgánicamente y/o acumulados y utilizados por una persona, familia o institución en el curso de sus actividades y funciones, así como todos aquellos documentos relevantes para la historia y desarrollo del país.?

En el cumplimiento de su misión el AN ofrece servicios en cada una de las cuatro sedes con que actualmente cuenta: Archivo Nacional Histórico (ANH), Archivo Nacional de la Administración (ARNAD), Archivo Regional de la Araucanía (ARA) y Archivo Regional de Tarapacá (ART).

El ANH es la sección del AN que resguarda la documentación creada desde la época colonial hasta las primeras décadas del siglo XX, además de colecciones particulares de distinta data, adquiridas o donadas a la institución (Archivo Nacional de Chile, s. f.-a).

La institución recibe a sus usuarios en su única sede, ubicada en la zona centro de Santiago. La consulta de documentos de forma presencial ocurre en la Sala de Lectura, espacio que cuenta con mesas de trabajo, terminales de consulta y reproductores de microfilm, además de un número considerable de índices y catálogos impresos, que siguen siendo uno de los principales medios de búsqueda de documentación entre los usuarios que acuden al archivo. Estos catálogos tienen diversas fechas de elaboración, datando algunos de ellos de principios del siglo XX. Por este motivo, solo una fracción reciente ha sido elaborada siguiendo estándares de descripción archivística (norma ISAD-G). En otros casos, pueden encontrarse una gran diversidad de formatos y criterios de organización y presentación de contenidos. ${ }^{8}$

Dependiendo del fondo documental que se consulte, los usuarios pueden utilizar los instrumentos de búsqueda disponibles en soporte papel o bien consultar alguna de las bases de datos del Archivo. Al momento de realizar esta investigación, el ANH contaba con tres plataformas de consulta diferentes: Aleph, WinISIS y Cyberdocs. Las dos primeras corresponden a software para descripción de recursos bibliográficos, mientras que la tercera fue desarrollada específicamente para el Archivo Nacional, siendo la única de las tres que permite la visualización de imágenes.

7. No obstante, al momento de la realización de este estudio la mencionada ley no se encontraba vigente. La misión del AN constaba en el DFL 5200 de 1929, que establecía en su artículo 13: "El Archivo Nacional tiene por objeto reunir y conservar los archivos de los departamentos de Estado y de todos los documentos y manuscritos relativos a la historia nacional, y atender a su ordenación y aprovechamiento".
8. Es importante acotar que los fondos del ANH no cuentan con una clasificación por serie documental. En cambio, han sido físicamente organizados en volúmenes, correspondientes a agrupaciones físicas de documentos empastados, lo que se ha manifestado también en términos de control intelectual, al crearse instrumentos que presentan descripciones por volumen y luego directamente a nivel de unidad documental (expediente o documento). 
9. El ANH también ofrece un servicio de referencia remota, que se realiza mediante correo electrónico.

10. Según la información entregada por la institución, solo recientemente se ha comenzado a sistematizar la información contenida en las papeletas. Al momento de realizar esta investigación, dicha información no estaba siendo utilizada por la institución para hacer un seguimiento de sus usuarios.

11. El ANH funciona de forma continuada de lunes a viernes entre 09:00 y 17:30 horas. La solicitud de volúmenes impresos se realiza en los siguientes horarios: 09:00 a 09:30, 11:00 a 11:30, 13:00 a 13:30 y 15:00 a 16:00 horas.

12. Así, el Reglamento de Usuarios establece que son usuarios permanentes "aquellas personas que hacen uso constante del material documental del $\mathrm{ANH}$, ya que este constituye un insumo para su desempeño profesional. En esta categoría están: doctores, magíster, doctorandos y académicos de trayectoria en el ámbito la historia, las letras y otras ciencias afines." Los usuarios temporales son "las personas que en razón de la actividad que realizan, harán uso de una documentación específica, durante un tiempo determinado. Es el caso de estudiantes de pregrado y

estudiantes de magíster. Asimismo,

están incluidas aquellos usuarios que se inician en las actividades de investigación." Por último, son usuarios ocasionales "aquellas personas que buscan indagar

solo respecto de una información específica, por una sola vez, y por tal motivo no concurrirán al ANH por tiempos prolongados."

13. Para todos los datos presentados, debe considerarse que las respuestas válidas no corresponden necesariamente al total de la muestra, sino que estas varían entre preguntas. Esto es una consecuencia de la aplicación de encuestas autoadministradas, mecanismo que no asegura que cada usuario complete la totalidad de las preguntas incluidas, o que se incurra en errores al completar el instrumento. Por este motivo, en todos los casos se señala el porcentaje en relación con las respuestas válidas, y no a los 93 casos que conforman la muestra.
En la Sala existe un mesón de atención de usuarios, donde un funcionario recibe las solicitudes de documentación y ofrece servicios de referencia cuando es requerido.? Para solicitar documentos, los usuarios deben completar una papeleta y presentarla en el mesón. ${ }^{10}$ Esta solicitud se puede hacer de forma continuada durante el horario de funcionamiento del archivo cuando se trata de microfichas, mientras que para volúmenes impresos existen solo cuatro horarios en los cuales se puede solicitar documentación (Archivo Nacional de Chile, s. f.-b). ${ }^{11}$

Toda la documentación del AN es de carácter público, por lo que puede ser libremente consultada por los usuarios. Como único requisito, cada visitante debe pasar por una acreditación, existiendo requisitos diferentes dependiendo del tipo de usuario. El ANH distingue entre tres tipos distintos: usuarios permanentes, usuarios temporales y usuarios ocasionales. La distinción entre unos y otros deriva de la frecuencia de uso de los servicios del archivo, asociándose de forma indirecta con la ocupación (Archivo Nacional de Chile, s. f.-c). ${ }^{12}$

\subsection{La experiencia de los usuarios del ANH}

La distinción que el ANH establece entre sus usuarios es el reconocimiento de la diversidad que puede encontrarse entre quienes acuden a este archivo. El grupo de usuarios que conforman la muestra de este estudio -considerando tanto a participantes de grupos focales como a encuestados- representa parcialmente esta diversidad, hecho que es relevante para el análisis de los resultados. Por una parte, puede observarse una notoria presencia de personas (66 en total) cursando estudios superiores y profesionales titulados, llegando en el grupo de encuestados a un $71,7 \%$ de las respuestas válidas ${ }^{13}$. En cuanto a las disciplinas o áreas del conocimiento con las que se vinculan los usuarios encuestados, a pesar de encontrar un rango relativamente amplio (Administración, Economía, Musicología, entre otras) se observa un predominio de personas que acuden al archivo para fines relacionados con la investigación histórica (74 personas, que constituyen el 82,2\% de las respuestas válidas) y, de forma bastante secundaria, con los estudios genealógicos (9 personas, equivalente al 10\%), proporción similar a la encontrada en los grupos focales. Por último, pueden observarse diferencias en términos de experiencia previa y frecuencia de uso del archivo, aunque se observa un predominio de usuarios experimentados, especialmente en los grupos focales, integrados en su mayoría por personas con más de un año de experiencia como usuarios de archivos de distinto tipo. En el caso de los encuestados, 57 personas (76\% de las respuestas válidas) de los encuestados acudió al ANH al menos una vez al mes en los seis meses previos, mientras que solo 9 accedían a los servicios ofrecidos en la Sala de Lectura del ANH por primera vez.

\subsubsection{Factores de mayor incidencia en la experiencia de los usuarios}

A la luz de los relatos de los usuarios, características tales como el nivel educacional, disciplina y experiencia previa en otros archivos, permiten comprender la forma en que ciertos grupos de usuarios se han relacionado con el archivo y su trayectoria específica dentro del ANH. En el caso del grupo estudiado, es posible advertir dos elementos clave que marcan la experiencia y trayectoria de los usuarios en el archivo: el acompañamiento de los pares y la guía de los funcionarios del archivo. La importancia dada a ambos factores es explicada por la novedad que representa el archivo en los primeros acercamientos y el desconocimiento de las dinámicas de funcionamiento y de los medios para explorar los conjuntos documentales que el ANH preserva. Los relatos de las primeras experiencias son ilustradores. Así, por ejemplo, comenta un usuario: 
me acuerdo de que el primer día (...) me fui conversando (...) con un compañero de la licenciatura, asustado con esta experiencia nueva. Y yo me acuerdo que estuve las tres horas con dos fojas y no avancé más. No entendía nada y estaba asustado.

En la misma línea, otro usuario señala que "las primeras veces (...) perdía el día, porque entre entender la dinámica interna de este espacio, saber identificar dónde tenía que buscar, las primeras veces fueron como...exploraciones."

De este modo, los usuarios reconocen que conocer el archivo y sus dinámicas es un proceso que toma tiempo, y que las habilidades para buscar información se desarrollan progresivamente. En este proceso, parece adquirir relevancia el acompañamiento de pares o guías que ayuden al nuevo usuario a familiarizarse con el espacio, sus fondos documentales y dinámicas de funcionamiento, como relatan aquellos usuarios que se iniciaron en el archivo a través de la realización de ejercicios iniciales de investigación o en visitas en el marco de cursos específicos como parte de sus estudios universitarios. En algunos casos, la relevancia de este tipo de vínculo se mantiene en el tiempo y se evidencia en la colaboración entre pares y entrega de datos de utilidad entre investigadores. No obstante, al contrastar estas apreciaciones con el grupo más amplio de usuarios encuestados, este tipo de acompañamiento parece ser importante, aunque en menor medida, declarándose como común en el 39,5\% de las respuestas válidas (30 casos).

Por otra parte, es destacado el rol que cumplen los funcionarios responsables de la atención de usuarios en la Sala de Lectura del ANH. En el relato de los usuarios, se transforma en un factor clave para comprender los instrumentos descriptivos e identificar conjuntos documentales de mayor relevancia para la investigación, pudiendo incluso determinar el éxito del trabajo de búsqueda documental. De esta manera, los usuarios reconocen que la experiencia puede variar según la persona que esté a cargo de la atención en un momento determinado, y se vislumbra el vínculo de confianza que se va desarrollando entre algunos de los usuarios y funcionarios, especialmente cuando los usuarios tienen más antigüedad en el ANH. De forma bastante gráfica, al referirse a uno de los encargados del servicio de referencia, un usuario relata: "yo me siento protegido cuando [él] está (...) porque él me orienta mucho en lo que tengo que hacer. Si no está (...) me siento medio así desamparado."

Del mismo modo, al comentar de forma abierta su experiencia en el ANH, es frecuente ver entre los encuestados la mención a la calidad de la atención y a los funcionarios que trabajan en la Sala destacando, en general, su amabilidad y disposición a brindar ayuda. Considerando que 82 personas (equivalentes al 94,3\% de las respuestas válidas) declaró haber realizado consultas a los funcionarios de la Sala para apoyar su búsqueda de documentos, estos atributos cobran valor.

Por otra parte, también puede verse que la experiencia de los usuarios está condicionada por las características de los instrumentos de consulta, la dinámica de solicitud de material que el ANH ha establecido y, en menor medida, por la infraestructura y equipamiento.

La valoración de los instrumentos de consulta fue un aspecto abordado extensamente en el diálogo entre usuarios, pudiendo contrastarse opiniones diversas. Por una parte, algunas personas manifiestan su satisfacción frente a los instrumentos de búsqueda de documentos que ofrece el ANH, mientras otras expresan una visión marcadamente crítica. Dentro del mismo diálogo, puede verse que estas discrepancias derivan tanto del grado de experiencia del usuario en un determinado fondo documental como de la variedad de instrumentos que ofrece el ANH. De esta manera, se reconoce que hay instrumentos descriptivos que tienen una calidad superior a otras por lo que, 
dependiendo del fondo que se consulte, pueden considerarse de mayor o menor calidad o utilidad para el ejercicio investigativo.

Aun considerando esta diferencia de opiniones, los usuarios ponen de relieve las deficiencias que han identificado en los instrumentos descriptivos, haciendo alusión principalmente a los índices y catálogos impresos que están disponibles en la Sala de Lectura. Dentro de las falencias identificadas está el nivel de detalle de las descripciones, que se considera, en muchos casos, insuficiente. Por otra parte, también se reporta que en ocasiones las descripciones no son representativas del contenido de las unidades de descripción, o que no han sido actualizadas a lo largo del tiempo, por lo que no contienen información sobre los ingresos de documentos más recientes.

Los usuarios también advierten un problema que afecta al conjunto de instrumentos: las diferencias que existen en términos de calidad y la inconsistencia entre los criterios utilizados en cada uno para organizar la información y describir el contenido. En palabras de uno de ellos:

el problema del [catálogo] físico es que cada vez que alguien desde los '70, o incluso de atrás en adelante, se decidía a hacer un catálogo de algún fondo, lo hacía 'a su pinta': lo hacía de acuerdo al abecedario, a fechas, a cómo iban apareciendo los documentos en los volúmenes. Entonces, es un tremendo dolor de cabeza empezar a revisar los catálogos, porque ninguno coincide con el otro, no hay una especie de orden del archivo tal cual, especificado y tipificado claramente, con el que uno pueda buscar los documentos.

También en relación con su contenido, comentan que, si bien se han hecho nuevas versiones de algunos catálogos, la información que entregan no es necesariamente la misma que constaba en los antiguos instrumentos de consulta, lo que en la práctica dificulta el rastreo de información previamente recolectada y la localización de documentos referenciados por otras investigaciones. Sobre esto, también se comenta que existen nuevos catálogos que utilizan terminología extemporánea en reemplazo de la terminología original, lo que aumenta las dificultades para acceder a la información.

Por último, algunos usuarios comentan que han tenido noticia de fondos que no han sido descritos, o bien que han sido descritos parcialmente, lo que los lleva a percibir que en el archivo hay mucho más de aquello a lo que ellos tienen acceso:

también [he tenido] esa idea de que se viene a conocer una ola gigante de información (...) aparece una cosa que te interesa acá y otra que te interesa por acá. Después encuentro otra cosa y al final uno quiere (...) tratar de hacerlo todo y después se da cuenta que es imposible (...) Hay cosas que no están ni siquiera catalogadas todavía, que ni siquiera existen dentro de los catálogos formales... [y] uno se pregunta: y a esta información ¿cómo podría acceder?

Otro punto que surge en el relato de los usuarios es la dificultad que puede generar la dinámica de funcionamiento del archivo, específicamente el sistema de horarios de solicitud que fue mencionado en el apartado anterior, que no es del todo comprendido por los usuarios.

Este es un elemento que, combinado con las características de los instrumentos descriptivos, puede extender más allá de lo necesario el tiempo que requiere la búsqueda de documentos. En este ámbito, los relatos de experiencias dan cuenta de cómo este sistema puede retrasar los procesos de investigación, especialmente para aquellos usuarios que no disponen de jornadas completas para acudir al archivo, sino que, 
por el contrario, solo disponen de sus horas de descanso para visitar el ANH. Como señala uno de ellos:

Al correr del tiempo uno muchas veces va teniendo otro tipo de responsabilidades, no solamente dedicando $100 \%$ a la investigación que sería lo ideal, pero en este país es bastante complejo hacerlo. Entonces tener que conciliar los tiempos muy marcados que tienen el archivo, con las labores que uno hace a nivel académico profesional, cada año sigue siendo complejo.

Por otra parte, se valora positivamente la existencia de un sistema de solicitud anticipada de documentos, que permite que los usuarios puedan requerir los volúmenes a consultar el día anterior a su visita, ayudando a sortear parcialmente las dificultades derivadas del sistema de horarios fijos y así reducir los tiempos de espera, al menos al comienzo de cada jornada.

Finalmente, pueden mencionarse algunas observaciones de los usuarios en términos de infraestructura y equipamiento. En este ámbito, surgen de forma espontánea, tanto en el diálogo de usuarios como en parte de los comentarios de los encuestados, alusiones a los problemas que puede generar el bajo número de estaciones de consulta y lectores de microficha disponibles, así como la falta de mantención de los mismos, o de tomas de corriente que permitan a los usuarios trabajar en sus propios equipos. No obstante, entre los participantes de los grupos focales, en general se valora positivamente la calidad de la Sala de Lectura, principalmente por ofrecer las condiciones apropiadas para la realización de investigación (iluminación, comodidad del mobiliario) y por el cuidado que los funcionarios ponen en el mantenimiento de una atmósfera silenciosa de trabajo. En relación con este aspecto, en los relatos de los usuarios se observa que la positiva valoración se desprende con frecuencia de la comparación que se establece entre la Sala de Lectura del ANH y las características de los espacios disponibles en otros archivos locales.

\subsubsection{Principales necesidades detectadas}

Tanto en los grupos focales como en las encuestas se exploraron las necesidades de los usuarios, mediante la identificación de aquellas áreas de mejora que podrían favorecer su experiencia en el ANH.

Al ser consultados sobre este punto, los participantes de los grupos focales mencionaron ámbitos que, en su mayoría, habían sido salido a la luz previamente y que, por lo tanto, se relacionan con las experiencias que han sido descritas en el apartado anterior. Las principales áreas que requieren mejoras desde el punto de vista de los usuarios son cuatro. Primero, la modificación de los horarios y modalidades de funcionamiento, lo que implicaría, idealmente, extender los días y horas de funcionamiento, y cambiar el sistema de horarios fijos para solicitud de documentos. Según sostuvieron algunos usuarios, esto contribuiría a democratizar el archivo, al permitir que usuarios de distintos perfiles, ocupaciones e intereses, pudiesen acceder con mayor facilidad y frecuencia a los servicios que ofrece el ANH. En segundo lugar, se mencionaron las mejoras en equipamiento, específicamente, en la cantidad de lectores de microfichas, el mantenimiento de los existentes y la mejora de la calidad de microfilmación de documentos. En tercer lugar, se discutió la necesidad de capacitar en mayor profundidad a funcionarios, especialmente a los auxiliares del archivo, lo que permitiría una experiencia consistente en el servicio de referencia, evitando las disparidades que varios de ellos observan en cuanto a la capacidad de los funcionarios de proporcionar información de utilidad. 
Finalmente, se observa un énfasis en la necesidad de avanzar en el ámbito tecnológico. La mayor parte de los usuarios visualiza el desarrollo de tecnologías como un medio para mejorar la calidad de los instrumentos descriptivos y facilitar su uso más allá de los límites físicos de la Sala de Lectura. La digitalización de catálogos y la disponibilidad de los mismos a través de la web son vistas como medidas que permitirían aumentar la accesibilidad a los documentos, reducir los tiempos de búsqueda y aumentar su efectividad. Uno de los usuarios sugiere una mejora en los mecanismos de control de la información, para que estos

permitan una mayor revisión de documentos, de los catálogos, de manera que podamos avanzar en investigaciones un poco más sofisticadas. (...) para muchos, muchos fondos, se requiere avanzar en ese tema. Que los usuarios tengamos un terminal de computador, al cual accedamos, por el cual pidamos los documentos, por el cual se registre la historia de la investigación y ese historial también nos permita acceder a documentos microfilmados o digitalizados de manera de evitar la movilidad de los documentos originales.

Las áreas que requieren mejoras también fueron abordadas en la encuesta, solicitándose a los participantes que identificaran cinco elementos prioritarios para la mejora de los servicios, ordenándolos por orden de prioridad. Como puede observarse en la Tabla 1, los elementos destacados fueron, en orden de mención: las plataformas de búsqueda y consulta de documentos; la cantidad de equipamiento tecnológico; el ambiente de trabajo en la Sala de Lectura; las herramientas no-informáticas de búsqueda de documentos, y finalmente, los días y horarios de funcionamiento.

\begin{tabular}{|l|c|}
\hline Área & $\begin{array}{c}\text { Número de } \\
\text { menciones }\end{array}$ \\
\hline Plataformas en línea de búsqueda y descripción de documentos & 56 \\
\hline $\begin{array}{l}\text { Cantidad de equipamiento tecnológico (computadores y lectores de } \\
\text { microficha) }\end{array}$ & 44 \\
\hline $\begin{array}{l}\text { Ambiente de trabajo en Sala de Lectura (iluminación, temperatura, } \\
\text { ruido ambiental) }\end{array}$ & 43 \\
\hline $\begin{array}{l}\text { Herramientas no-informáticas de búsqueda y descripción de } \\
\text { documentos (catálogos impresos, guías, índices, etc.) }\end{array}$ & 41 \\
\hline Conveniencia de días y horarios de funcionamiento & 37 \\
\hline Disponibilidad de espacios de trabajo en Sala de Lectura & 36 \\
\hline Estado físico de los documentos (volúmenes y microfichas) & 34 \\
\hline $\begin{array}{l}\text { Calidad de servicios de solicitud y entrega de materiales (volúmenes } \\
\text { y microfichas) }\end{array}$ & 32 \\
\hline $\begin{array}{l}\text { Calidad de servicios de orientación y ayuda a usuarios/as en Sala de } \\
\text { Lectura }\end{array}$ & 31 \\
\hline $\begin{array}{l}\text { Calidad de espacios públicos disponibles (áreas de tránsito, descanso, } \\
\text { baños, etc.) }\end{array}$ & 20 \\
\hline Calidad de la atención a usuarios/as del archivo en recepción del ANH & 17 \\
\hline Calidad de servicios de reproducción ofrecidos en el archivo & 15 \\
\hline Accesos al edificio & 12 \\
\hline Calidad servicios de certificación ofrecidos en el archivo & 5 \\
\hline
\end{tabular}


Como puede observarse, en general, existe coincidencia entre los elementos que fueron nombrados como prioritarios por los encuestados y aquellos identificados por los participantes de los grupos focales, a excepción del ambiente de trabajo de la Sala de Lectura, elemento positivamente valorado en las instancias de discusión, y la capacitación a funcionarios, que no fue un elemento sometido a evaluación en la encuesta.

\subsection{Satisfacción general de usuarios del ANH}

Las experiencias y percepciones presentadas en las secciones anteriores permiten hacer algunas inferencias en torno a la satisfacción general de los usuarios del ANH incluidos en este estudio.

Como primer punto, puede mencionarse que los usuarios manifestaron mayoritariamente estar satisfechos con su experiencia en el ANH. En la encuesta, los participantes tuvieron la posibilidad de evaluar distintos ámbitos de los servicios del archivo, manifestando su satisfacción en una escala de 1 a 7 puntos. ${ }^{14}$ Descontando la ubicación y accesos al edificio, que recibieron las más altas evaluaciones, destacan en primer lugar, paradojalmente los servicios de solicitud y entrega de materiales, aspecto que había sido descrito como problemático en las instancias de discusión ${ }^{15}$. A continuación, siguen la atención a usuarios en la recepción del ANH; la comodidad de los espacios disponibles; los servicios de orientación y ayuda en Sala y, finalmente, la disponibilidad de espacios de trabajo en la Sala de Lectura.

Por otra parte, están aquellos aspectos que generan menor satisfacción. Encontramos en la posición más baja la calidad del equipamiento tecnológico, promediando 4,6. En orden ascendiente, le siguen: la cantidad de equipamiento tecnológico; la facilidad de uso de plataformas en línea para consulta de documentos; la utilidad de dichas plataformas, y el estado físico de los documentos. En cuanto a la facilidad de uso de instrumentos descriptivos impresos, aspecto ampliamente comentado en los grupos focales, estos se ubicaron unos peldaños más arriba de los elementos más críticos, con un puntaje de 5,7. Como puede observarse, los ámbitos de mayor satisfacción se vinculan a la calidad de los servicios e infraestructura de apoyo, mientras que los de menor satisfacción se vinculan principalmente a los medios disponibles para búsqueda de documentos, principalmente aquellos de tipo informático. La lista completa de puntajes puede observarse en la Tabla 2.

Respecto a la experiencia general el día de la visita a la Sala de Lectura, la valoración de los usuarios fue positiva, promediando 6,3. Al preguntarse de forma abierta por los motivos tras el nivel de satisfacción, se observó una tendencia a destacar la calidad humana, cordialidad y amabilidad por parte de los funcionarios, así como la comodidad del espacio, aspectos que, como se ha visto, también fueron positivamente valorados en los grupos focales. También se enfatiza la rapidez de la entrega de materiales cuando estos son solicitados con antelación, aunque se manifiestan algunas opiniones contrapuestas que manifiestan reparos por los tiempos de espera bajo el sistema de horarios fijos, sin que impacte mayormente el nivel general de satisfacción del grupo de encuestados.
14. La escala de 1 a 7 fue utilizada puesto que es la escala que por disposición legal se utiliza en todos los niveles educacionales en Chile, por lo cual podía resultar familiar a cualquier usuario nacional del ANH. En esta escala, se considera que el mínimo de aprobación es 4.

15. Es probable que esta contradicción se deba a que los usuarios estén evaluando otros ámbitos dentro del proceso de solicitud y entrega de materiales, sin considerar la dinámica de solicitud en horarios fijos establecida por el $\mathrm{ANH}$. 
Tabla 2: Evaluación de instalaciones, servicios y búsqueda y consulta de documentos en el ANH

\begin{tabular}{|c|c|}
\hline Ámbito & $\begin{array}{c}\text { Satisfacción } \\
\text { promedio (1-7) }\end{array}$ \\
\hline \multicolumn{2}{|l|}{ Instalaciones } \\
\hline Ubicación & 6,72 \\
\hline Accesos al edificio & 6,66 \\
\hline $\begin{array}{l}\text { Comodidad de los espacios públicos disponibles (áreas de tránsito } \\
\text { y descanso, baños, etc.) }\end{array}$ & 6,14 \\
\hline Disponibilidad de espacios de trabajo en Sala de Lectura & 5,96 \\
\hline $\begin{array}{l}\text { Ambiente de trabajo en Sala de Lectura (iluminación, temperatura, } \\
\text { ruido ambiental) }\end{array}$ & 5,86 \\
\hline $\begin{array}{l}\text { Cantidad de equipamiento tecnológico (computadores y lectores } \\
\text { de microficha) }\end{array}$ & 5,01 \\
\hline $\begin{array}{l}\text { Calidad del equipamiento tecnológico (computadores y lectores } \\
\text { de microficha) }\end{array}$ & 4,56 \\
\hline \multicolumn{2}{|l|}{ Servicios de apoyo } \\
\hline $\begin{array}{l}\text { Calidad de servicios de solicitud y entrega de materiales } \\
\text { (volúmenes y microfichas) }\end{array}$ & 6,17 \\
\hline $\begin{array}{l}\text { Calidad de la atención a usuarios/as del archivo en recepción del } \\
\text { ANH }\end{array}$ & 6,15 \\
\hline $\begin{array}{l}\text { Calidad de servicios de orientación y ayuda a usuarios/as en Sala } \\
\text { de Lectura }\end{array}$ & 6,08 \\
\hline Calidad de servicios de reproducción ofrecidos en el archivo & 5,92 \\
\hline Calidad servicios de certificación ofrecidos en el archivo & 5,81 \\
\hline Conveniencia de días y horarios de funcionamiento & 5,51 \\
\hline \multicolumn{2}{|l|}{ Búsqueda y consulta de documentos } \\
\hline $\begin{array}{l}\text { Utilidad de herramientas no-informáticas de búsqueda y } \\
\text { descripción de documentos (índices, guías o catálogos impresos) }\end{array}$ & 5,89 \\
\hline $\begin{array}{l}\text { Facilidad de uso de herramientas no-informáticas de búsqueda y } \\
\text { descripción de documentos (índices, guías o catálogos impresos) }\end{array}$ & 5,7 \\
\hline Estado físico de los documentos (papel y microfichas) & 5,32 \\
\hline $\begin{array}{l}\text { Utilidad de plataformas en línea de búsqueda y descripción de } \\
\text { documentos }\end{array}$ & 5,14 \\
\hline $\begin{array}{l}\text { Facilidad de uso de plataformas en línea de búsqueda y } \\
\text { descripción de documentos }\end{array}$ & 5,06 \\
\hline
\end{tabular}

Estas impresiones se replicaron en los grupos focales, en los que los usuarios manifestaron mayoritariamente estar satisfechos con los servicios provistos por el ANH, aun considerando las críticas esbozadas. El énfasis está nuevamente en el servicio entregado por las personas que trabajan en la Sala de Lectura, destacándose su voluntad y esfuerzo por ofrecer servicios de calidad a pesar de las limitaciones percibidas por los mismos usuarios. En este sentido, como menciona uno de los usuarios, destaca "el esfuerzo que hacen los funcionarios (...) porque tienen una disposición (...) y si no está en el catálogo uno conversa con ellos y siempre te dan una idea de por dónde puede uno ir". Añade otro usuario que, a pesar de la aparente falta de recursos, los funcionarios "logran hacer muchas cosas que nos facilitan la vida a muchos y muchas". 
Por otra parte, también se comenta y valora positivamente el trabajo que ha hecho el ANH en los últimos años por abrir espacios a la comunidad y difundir su trabajo, así como por mejorar los servicios que se ofrecen en la Sala de Lectura:

el archivo cumple su rol también en la medida, en que, me parece, hay más personas interesadas en venir a investigar el material que hay acá. El archivo depende tremendamente de estudiantes, investigadoras, historiadoras, historiadores. Creo que (...) ha aumentado la población de historiadores, historiadoras, y creo que eso también ha alimentado, ha nutrido que las funciones del archivo se pulan, hayan mejorado, se hayan reestructurado.

Finalmente, cabe mencionar que, en el desarrollo de estas discusiones, este reconocimiento y respaldo generalizado a la labor del ANH en su conjunto, contrasta con algunas impresiones más críticas sobre el rol del Estado chileno, al manifestarse dudas de su interés en este ámbito y discutirse la responsabilidad que tiene en la asignación de recursos. Es este sentido, parte de los usuarios que participó en la discusión ve en este hecho el argumento que explica la capacidad limitada del ANH de mejorar las condiciones materiales en que ofrece sus servicios.

\section{Discusión}

Los resultados presentados pueden situarse en dos dimensiones diferentes: primero, aquella vinculada a la experiencia de los usuarios en el acceso a servicios en el ANH, dentro de la cual puede discutirse la relevancia que tienen ciertos factores y posibles perspectivas para su abordaje. Segundo, la metodológica, en la que se puede comentar la idoneidad y aporte de las técnicas utilizadas y la forma en que contribuir al estudio de la experiencia de los usuarios de archivos.

\subsection{La experiencia de los usuarios del ANH}

En relación con el primer punto y a la luz de los resultados, es posible observar que existen cuatro factores principales incidiendo en la experiencia de los usuarios del ANH: la infraestructura y equipamiento disponibles; las condiciones en que se ofrecen los servicios, incluidos los procedimientos establecidos por la institución; los instrumentos descriptivos y herramientas que permiten la búsqueda de información, y el desempeño de las personas que actúan como intermediarios entre usuarios e información/documentación.

El primero, como hemos visto, se refiere principalmente a las condiciones que propician el trabajo en la Sala de Lectura, considerando principalmente el espacio disponible, la cantidad y calidad de equipos de búsqueda y consulta documental, e incluso las condiciones ambientales. Más allá de las diferencias expresadas en la valoración individual de cada aspecto, este ámbito emerge de forma recurrente en el relato de los usuarios, dando cuenta del peso que tiene dentro de su experiencia. De hecho, este factor ha sido identificado previamente en otros estudios como uno de los atributos que permiten evaluar la calidad en los archivos (Ladrón de Guevara, 2010). De todos los aspectos, el equipamiento es probablemente el más objetivo y medible, estando su mejora directamente vinculada con la disponibilidad de recursos económicos por parte del archivo. No ocurre lo mismo al considerar los otros tres factores - condiciones del servicio, instrumentos y herramientas descriptivas y rol de los intermediarios-, siendo necesario el despliegue de una mayor cantidad y variedad de recursos para la identificación de puntos críticos e implementación de mejoras. 
De la información presentada puede inferirse que estos tres factores están relacionados. Aunque no contamos con la evidencia suficiente aún para poder determinar de forma más precisa de qué manera cada uno está incidiendo sobre el otro, puede esbozarse como escenario posible que la dificultad de los usuarios para comprender y utilizar las plataformas de consulta y los instrumentos descriptivos provistos por el archivo puede estar generando una sobre demanda de los servicios de referencia. En otras palabras: puesto que los usuarios tienen dificultades para navegar la "ola de información" -como uno de ellos la ha descrito- de forma autónoma, requieren con frecuencia de la orientación de uno de los funcionarios a cargo del servicio de referencia. De acuerdo a lo señalado por los investigadores, la orientación ofrecida puede variar de forma significativa dependiendo del funcionario a cargo, lo que significa que no necesariamente se conseguirá un resultado exitoso. Luego, el tiempo y esfuerzo invertido en el proceso de búsqueda, tanto por parte de los usuarios como de los trabajadores del archivo, puede aumentar de forma notoria cuando se requieren volúmenes que, debido al sistema de horarios de solicitud, no podrán ser entregados de forma inmediata, dando lugar a los tiempos de espera que describen los usuarios.

Determinar si la relación entre estos factores es efectiva sería una forma de canalizar los esfuerzos de la institución hacia aquellas áreas que realmente lo necesitan (ya sea la capacitación de funcionarios, la mejora de las plataformas e instrumentos descriptivos, el cambio de las dinámicas de solicitud, o la combinación de estas). Si efectivamente existe la sobrecarga en el servicio de referencia que describen los usuarios, el riesgo está en la sostenibilidad del servicio en el nivel actual. Siendo la atención de los funcionarios, por lejos, el ámbito más destacado y valorado por los usuarios del ANH, cabe preguntarse si acaso la alta satisfacción general descansa mayormente sobre dicha atención. Si así fuera, cualquier cambio en el mediano o largo plazo en los funcionarios a cargo podría poner en riesgo los niveles de satisfacción que, según este estudio, el ANH ha logrado alcanzar.

A la hora de estudiar cada uno estos cuatro ámbitos, puede ser relevante considerar su inscripción dentro de lo que Margaret Hedstrom denominó, hace algunos años, como "interfaz archivística" (archival interface). La autora utiliza este concepto tanto como una metáfora sobre el rol de los archivistas como intermediarios entre la evidencia documental y sus lectores, como un término que describe el conjunto tangible de estructuras y herramientas que sitúan a los documentos de archivo en un contexto y proveen un marco interpretativo (Hedstrom, 2002: 22).

16. Esta visión es coincidente con la de otros autores, que conciben al archivista "como un actor, no un guardián; un representador, no un custodio" (Cook y Schwartz, 2002: 183). El rol mediador del archivista no ha sido reconocido como un elemento negativo per se, sino que solo en la medida en que, según se sostiene, no se ha desarrollado un suficiente nivel de conciencia sobre el mismo, y sobre la relación de poder que dicho rol mediador conlleva, dando paso a la naturalización de prácticas sin mayor reflexión. De ahí que el énfasis de estos y otros autores está en que las instituciones archivísticas adquieran conciencia sobre su propio poder y de cómo mediante sus prácticas permiten o limitan el acceso a la información y, por consiguiente, a la interpretación del pasado (Hedstrom, 2002: 22).
Como señala la autora, esta interfaz es construida -de forma más o menos consciente- a través del conjunto de decisiones que se toman en todo ámbito del actuar archivístico, desde la elección de criterios de selección hasta las formas y plataformas escogidas para representar la información, lo que sitúa al archivo y su accionar en el centro, al reconocer el rol y responsabilidad que le cabe como mediador en el acceso a la información. ${ }^{16}$

¿Cómo se ha llegado a conformar la interfaz que opera entre el ANH y sus usuarios? No es posible establecerlo en esta instancia, pero podría determinarse estudiando las razones que subyacen en la definición de las dinámicas de funcionamiento, la forma en que se ha capacitado a los funcionarios, o los criterios que se han seguido para seleccionar, organizar y representar la documentación ante los usuarios en diversos instrumentos y plataformas. En cambio, las experiencias y percepciones aquí presentadas -al igual que las que puedan ser arrojadas por otros estudios de usuarios- sí permiten explorar los componentes y características de la interfaz, la forma en que es percibida por los usuarios, y la medida en que puede incidir en su satisfacción, al entender la Sala de Lectura como uno de los nodos más relevantes de encuentro y comunicación entre el ANH y sus usuarios. 
Entendiendo que la interfaz archivística permite la interacción de dos actores -el archivo y sus usuarios- resulta interesante abordar las acciones de mejora no solo a partir de cambios en el propio Archivo, sino que también considerando la participación de los propios usuarios. En este sentido, existen estudios que han puesto el énfasis en la "alfabetización archivística" como elemento central para el éxito en la experiencia de los usuarios. Este concepto se refiere a

el conocimiento del investigador de principios, prácticas e instituciones archivísticas, tales como las razones que subyacen a las reglas y procedimientos archivísticos, el cómo desarrollar estrategias para explorar preguntas de investigación, y la comprensión de la relación entre fuentes primarias y sus representaciones (Yakel y Torres, 2003: 52).

Desde la perspectiva de la alfabetización archivística, lo que distingue a los usuarios expertos de los usuarios con menor manejo del archivo (y presumiblemente a los más exitosos de los que encuentran más dificultades en su experiencia) es el grado en que conocen y están familiarizados con estos ámbitos. Así, por ejemplo, bajo esta mirada, un "usuario alfabetizado" debería comprender los principales conceptos y principios de la teoría archivística y las características de los principales instrumentos descriptivos. Esto le otorgaría mayor capacidad de satisfacer sus necesidades de información y, a la vez, comunicarse de forma efectiva con el referencista cuando sienta que sus propios conocimientos no son suficientes. ${ }^{18}$

A modo de ejemplo, podemos tomar lo que denominamos en un comienzo como "condiciones y procedimientos de consulta." Yakel y Torres han utilizado un concepto diferente para referirse al mismo factor, denominándolas como "reglas archivísticas." Estas reglas son aquellas que "afectan directamente la capacidad de hacer investigación y con frecuencia interrumpen patrones y rutinas de investigación de larga data" (Yakel y Torres, 2003). No obstante, las reglas archivísticas pueden ser aprendidas por los usuarios hasta el punto de volverse rutinarias, permitiendo que eventualmente estos puedan dedicar sus energías a su investigación, más que a la comprensión de las reglas que condicionan su búsqueda de información. Estas ideas tienen un paralelo con las experiencias de los usuarios en relación con las condiciones del servicio y dinámicas de solicitud que, en virtud de lo ya expuesto, son un factor a considerar en el retraso que puede presentarse en la búsqueda de fuentes documentales. Por otra parte, Yakel y Torres han señalado que el desarrollo de la alfabetización archivística es un proceso continuo, aún para los usuarios más experimentados, lo que coincide con los relatos de los usuarios sobre el tiempo como un factor relevante en la internalización de dichas reglas -en ocasiones gracias al apoyo de sus pares y de los trabajadores de la Sala de Lectura- lo que les ha permitido adaptarse gradualmente.

A pesar de que esta visión hace partícipes a los usuarios en la mejora de su experiencia, también asigna responsabilidad al propio archivo, aunque no de forma exclusiva. Esto, ya que la formación de usuarios puede ser complementada desde la educación universitaria en ciertas disciplinas más propensas a la investigación con fuentes primarias, como la Historia. Las acciones que el propio archivo puede tomar en esta materia han sido abordadas también en los trabajos ya citados sobre estudios de usuarios, existiendo autores que han advertido que la información recabada puede llevar a la creación de programas de formación de usuarios, de modo de generar instancias para el desarrollo de las competencias necesarias para la exploración de los archivos (Allendez Sullivan y de la Puente, 2013; Casado, 1993; Jaén García, 2006). En una línea similar, también existen estudios que han sugerido la mejora de la experiencia de los usuarios mediante la alfabetización de los propios archiveros que trabajan en servicios de referencia. Este tipo de alfabetización se orienta tanto al conocimiento del propio archivo,
17. El concepto originalmente acuñado por Yakel y Torres en 2003 fue "inteligencia archivística”, cuya definición se ha presentado. En 2016 Yakel y Malkmus utilizaron la misma definición, pero esta vez bajo el rótulo "alfabetización archivística", que para entonces ya se encontraba en uso en la literatura del área, razón por la cual también ha sido preferida en este texto.

18. Por otra parte, al menos en el ámbito del lenguaje, se puede cuestionar si es el usuario quien debe alfabetizarse archivísticamente o es el archivo quien debe adaptar lenguaje técnico al de sus usuarios. El discurso sobre alfabetización se entiende mejor en el contexto de los archivos históricos, que suelen tener usuarios por largos periodos de tiempo, pero en archivos administrativos esta situación puede variar drásticamente. Podemos preguntarnos: ¿Debiera un usuario ocasional manejar la teoría y terminología archivística? 
como al de los ámbitos que son relevantes desde la perspectiva del usuarios, incluyendo aspectos tales como el conocimiento del proceso de investigación, de los mecanismos de interacción (instituciones y prácticas, sistemas de acceso, técnicas de búsqueda, instrumentos de acceso, habilidades de comunicación, comprensión de comportamientos de búsqueda de información, por nombrar algunos) y conocimiento de las colecciones o conjuntos de documentos (Duff; Yakel y Tibbo, 2013).

Como puede suponerse a partir de lo expuesto, los ámbitos en los que un archivo como el ANH puede actuar incluyen la revisión de sus prácticas, procedimientos y, en general, de la forma en que se ofrecen los servicios de consulta de documentos, pero no están limitados a ello. Al considerar otras experiencias en el estudio de los usuarios se puede desprender que la mejora en su experiencia puede incorporar acciones que incluya y haga partícipes a los propios usuarios, además de otros actores relevantes, como son los propios trabajadores a cargo del servicio de referencia.

\subsection{Reflexiones metodológicas}

Dejando a un lado estas perspectivas para el abordaje y mejora de la experiencia de los usuarios, podemos discutir la dimensión metodológica de esta investigación. Si bien se considera satisfactorio el uso de las dos técnicas utilizadas de forma complementaria, este ejercicio también ha dado cuenta de sus limitaciones. Por una parte, los resultados de la encuesta no han podido ser utilizados en su máximo potencial (desde una perspectiva cuantitativa), dadas las características de la muestra. Aun así, la aplicación de este instrumento ha aportado información relevante para el conocimiento de parte de los usuarios del ANH y ha servido para contrastar la información recabada en los grupos focales dentro de un grupo de usuarios más amplio y diverso. En la mayor parte de los casos, se han reforzado tendencias (por ejemplo, en relación con las inquietudes frente a instrumentos descriptivos y el desarrollo tecnológico del archivo) y, en otros, se han manifestado matices que merecen una exploración más detallada. Por otra parte, los grupos focales han permitido recopilar una cantidad importante y valiosa de información en un grado de riqueza suficiente para conocer las experiencias y las dimensiones que adquieren mayor relevancia en la satisfacción de los usuarios participantes. Considerando que la satisfacción representa tanto las respuesta intelectuales, materiales y emocionales de los usuarios frente a sus necesidades de información (Hernández Salazar, 2011: 362), el uso de técnicas cualitativas que permitan la expresión abierta y en profundidad de las mismas es de gran valor. Sin embargo, también se pudieron advertir ciertos sesgos en el uso de esta técnica.

En el caso particular del ANH, el grupo de investigadores que participó en los grupos focales no permite captar la total diversidad de usuarios del archivo, aunque sí se representaron los dos grupos más importantes en términos de número: historiadores y genealogistas. Como es posible anticipar, el tiempo requerido para involucrarse en esta actividad limita la capacidad de participar de otras personas, tales como ciudadanos comunes que acuden al ANH a solicitar documentos específicos para la realización de trámites (tipificados por la institución como usuarios ocasionales), o profesionales que no tienen la investigación en el centro de su quehacer, y que por lo mismo cuentan con tiempo limitado para cada visita (por ejemplo, profesores de escuela). El resultado es que resulta difícil conocer, a través de una instancia como esta, la experiencia y visión de estos otros usuarios, que podría distar significativamente de la de los grupos con mayor experiencia que, dados sus largos tiempos de estadía en el archivo, tienen mayor experiencia y dominio del archivo y que pueden establecer vínculos de mayor profundidad con los funcionarios o tener acceso a información que no necesariamente tendría un usuario común. Estos usuarios sí pueden ser estudiados mediante el uso de 
otras técnicas que pueden favorecer una mayor cobertura, como ocurrió en esa investigación con la aplicación de encuestas. Dado lo anterior, al concluir este ejercicio exploratorio, es posible dar cuenta de los beneficios que implica la combinación de técnicas mixtas para el estudio de los usuarios de archivos, en tanto permite complementar, explicar y problematizar los resultados que podría arrojar un único instrumento o instancia.

A pesar de los límites declarados, en esta investigación se ha recabado información nueva, tanto para la institución como para su entorno, y que puede ser relevante para la evaluación y mejora de los servicios, especialmente en ausencia de fuentes de información indirectas, como por ejemplo, registros detallados de usuarios, sus perfiles, el uso que hacen de la totalidad de servicios provistos por la institución y sus consultas realizadas. ${ }^{19}$ La obtención de información con mayor nivel de precisión en el futuro estará condicionada por las mejoras que la institución pueda implementar en los métodos y procedimientos de captura de información sobre usuarios y usos del archivo. El contar con datos suficientes y de calidad, necesarios para hacer un análisis estadístico de la cuestión, permitirá que los beneficios del estudio de los usuarios de archivos que han sido descritos en un comienzo puedan, finalmente, concretarse.

\section{Conclusiones y recomendaciones}

Esta investigación ha permitido realizar una primera exploración en el ámbito de la experiencia de los usuarios de archivos, específicamente de aquellos que consultan de forma presencial los documentos preservados por el Archivo Nacional Histórico de Chile. En el ámbito metodológico, se puede concluir que el uso de instrumentos que permitan el estudiar la experiencia de los usuarios desde una perspectiva cuantitativa es de gran valor para establecer prioridades en la realización de acciones de mejora. Por otra parte, el uso del grupo focal ha permitido demostrar el aporte de esta técnica en la exploración en profundidad de experiencias particulares que, dadas sus características, son mejor comprendidas bajo una perspectiva cualitativa. Aunque se han identificado algunos sesgos y límites metodológicos que es importante considerar, el uso de técnicas mixtas de investigación ha permitido incorporar y contrastar información sobre la experiencia, necesidades y satisfacción de un grupo diverso de usuarios del ANH.

La triangulación de la información recabada en grupos focales y en la aplicación de encuestas, ha permitido identificar los cuatro ámbitos de mayor relevancia en la experiencia de los usuarios: la infraestructura y equipamiento disponibles; las condiciones en que se ofrecen los servicios, incluidos los procedimientos establecidos por la institución; los instrumentos descriptivos y herramientas que permiten la búsqueda de información, y el rol de las personas que actúan como intermediarios entre usuarios e información/documentación. Se identificaron las principales mejoras en cada ámbito que, desde la perspectiva de los usuarios, haría más satisfactoria su experiencia en el $\mathrm{ANH}$, siendo las áreas de mayor consenso la mejora de las plataformas de búsqueda y consulta de documentos, la disponibilidad del equipamiento necesario para la revisión de documentos, la calidad de los instrumentos descriptivos y la ampliación de los días y horas de funcionamiento.

$\mathrm{Al}$ abordar la satisfacción de los usuarios, se puede concluir que, en términos generales, los usuarios del ANH que participaron de este estudio, aún tras expresar algunas inquietudes y disconformidades, valoran de forma positiva los servicios ofrecidos por la institución. En particular, ha podido observarse que uno de los aspectos más valorados es la calidad de la atención en la Sala de Lectura, destacando los atributos
19. Sobre este punto, cabe mencionar que en la reunión de presentación de resultados y retroalimentación sostenida con autoridades y trabajadores del $\mathrm{ANH}$, se manifestó que, a pesar de las limitaciones declaradas, los resultados se alinean con sus percepciones tras años de trabajo en la institución. Pese a esta favorable coincidencia, vale reforzar la necesidad de contar con mayor evidencia que permita transitar desde la intuición a la certeza, al menos en términos de evaluación de la efectividad de los servicios ofrecidos. 
de los funcionarios del archivo y el rol que juegan en el acompañamiento y desarrollo de habilidades de los usuarios.

En relación con los ámbitos más relevantes para la experiencia de los usuarios, se han identificado dos formas complementarias de abordaje: una, desde el análisis y mejora individual de los servicios y productos generados por el archivo (entendidos como parte de la interfaz que el ANH ha diseñado para vincularse con sus usuarios) que, por ejemplo, podría traducirse en la mejora de las herramientas y sistemas de representación o descripción de información. La otra, se refiere al trabajo en el ámbito de la alfabetización archivística de los usuarios (e incluso de los responsables del servicio de referencia), lo que ayudaría en la comprensión de las dinámicas y servicios del ANH, la mejor comunicación entre los actores involucrados y el dominio de las habilidades necesarias para tener una experiencia satisfactoria en la búsqueda y consulta de documentos. Esto no solo dotaría a los usuarios de mayores niveles de autonomía, sino que también podría evitar o reducir la sobrecarga en los servicios de referencia que puede estar ocurriendo en el ANH.

Se sugiere enérgicamente implementar cambios en las formas de recopilar datos por parte de la institución, lo que permitiría incorporar a futuros estudios el análisis de fuentes indirectas de información, claves para el conocimiento de los usuarios. Esto permitiría establecer de forma más clara y precisa la tipología y perfil de usuarios de este archivo, lo que en el futuro posibilitaría un análisis más detallado de la experiencia y las necesidades de conglomerados específicos de usuarios. A la vez, podrían anticiparse nuevas demandas y mantener la positiva valoración general de los usuarios que día a día acuden de forma presencial al ANH para realizar sus investigaciones.

Por consiguiente, emerge naturalmente como línea de investigación el estudio de los propios usuarios del ANH, utilizando nuevas fuentes y tipos de datos, con los beneficios y usos que ya se han mencionado. Esto sin duda facilitaría que el archivo cumpla cabalmente con su principio ontológico, satisfaciendo las necesidades de información de sus usuarios.

20. Sobre esto, Richard Cox (2008) y otros archivistas han advertido en los últimos años que no se han realizado los ajustes necesarios para adaptar las prácticas descriptivas a las necesidades y hábitos de los usuarios.
En un ámbito más general, también pueden enunciarse algunas líneas. Por una parte, existe aún un vacío de información en cuanto al estudio y evaluación pormenorizada de los instrumentos descriptivos en uso en nuestros archivos. La investigación en este ámbito no solo ayudaría a asegurar que estos instrumentos sean efectivos, sino que también permitiría que los cambios tecnológicos e implementación de nuevos estándares -que indudablemente vendrán- avancen en la dirección correcta. Estas evaluaciones necesariamente deben estar centradas en la experiencia de los usuarios, y no en las percepciones o necesidades de los archivos. ${ }^{20}$ Por otra parte, puede añadirse que es necesaria la realización de nuevos estudios en otros archivos, especialmente dentro del ámbito latinoamericano. El conocimiento sobre los usuarios de nuestros archivos es escaso y disperso, y la elaboración de diagnósticos institucionales y regionales podría ayudar a orientar los estudios de usuarios y propuestas de mejora hacia las áreas que requieran mayor atención y que puedan, potencialmente, ser trabajadas de forma colectiva o colaborativa entre instituciones archivísticas. 


\section{Agradecimientos}

Agradezco de forma especial al Archivo Nacional Histórico de Chile, a su directora Dra. Emma de Ramón y a todos quienes trabajan en la institución, tanto por acoger este estudio y dar las facilidades necesarias para su ejecución, como por los valiosos comentarios recibidos al momento de presentar sus resultados. También agradezco a todos quienes generosamente dedicaron su tiempo para participar en esta investigación, especialmente a aquellos investigadores e investigadoras que sacrificaron parte de su jornada de trabajo para relatar sus experiencias y compartir sus reflexiones en los grupos focales realizados.

Por último, agradezco inmensamente el trabajo de Kassandra Alarcón y Claudio Rojas, quienes se desempeñaron como ayudantes de investigación. Su entusiasmo, dedicación y rigurosidad en el trabajo contribuyeron de forma significativa a la concreción de los objetivos planteados. 


\section{Q Referencias Bibliográficas}

"Acosta Sandoval, S. E.; E. M. Íncer Solís y A. Mena Aguilar. 2006. Propuesta teórica - metodológica para evaluar los servicios de los archivos a partir de un estudio de usuarios. Unidad de análisis: los archivos municipales. San José: Universidad de Costa Rica.

" Allendez Sullivan, P. y M. de la Puente. 2013. Los estudios de usuarios en los archivos. Buenos Aires: Consultora de Ciencias de la Información. <http://www. ccinfo.com.ar/v2/wp-content/uploads/2016/01/DT_047.pdf > [Consulta: 2 diciembre 2017].

»Archivo Nacional de Chile. (s. f.-a). Archivo Nacional Histórico. <http://www.archivonacional.cl/616/w3-article-80oo.html> [Consulta: 2 diciembre 2017].

»Archivo Nacional de Chile. (s. f.-b). Preguntas frecuentes. <http://www.archivonacional.cl/616/w3-propertyvalue-37351.html> [Consulta: 2 diciembre 2017].

"Archivo Nacional de Chile. (s. f.-c). Reglamento de usuarios (as) Archivo Nacional Histórico. <http://www.archivonacional.cl/616/articles-75846_recurso_01.pdf> [Consulta: 2 diciembre 2017].

"Calva González, J. J. 2004. La investigación sobre las necesidades de información en comunidades de usuarios. En Investigación Bibliotecológica. Vol. 18, no. 37, 23-55.

" Campos Ramírez, J. 2009. Los estudios de usuarios y los archivos: una alianza estratégica. En Revista Códice. Vol. 5, no. 1, 13-37.

"Congreso Nacional de Chile. 2017. Ley 21045. Creación del Ministerio de las Culturas, las Artes y el Patrimonio. <http://bcn.cl/22iar> [Consulta: 2 diciembre 2017].

»Cook, T. y J. M. Schwartz. 2002. Archives, Records, and Power: From (Postmodern) Theory To (Archival) Performance. En Archival Science. Vol. 2, no. 3-4, 171-185. <https://doi.org/10.1007/BFo2435620> [Consulta: 2 diciembre 2017].

»Cox, R. J. 2008. Revisiting the Archival Finding Aid. En Journal of Archival Organization. Vol. 5, no. 4, 5-32. <https://doi.org/10.108o/15332740802153245> [Consulta: 2 diciembre 2017].

"Decreto con Fuerza de Ley no. 5200. 1929. Sobre instituciones nacionales patrimoniales dependientes del Servicio Nacional del Patrimonio Cultural. Valparaíso: Congreso Nacional de Chile. <http://bcn.cl/1vaqb> [Consulta: 2 diciembre 2017].

»Duff, W.; E. Yakel y H. Tibbo. 2013. Archival Reference Knowledge. En The American Archivist. Vol. 76, no. 1, 68-94.

»Hedstrom, M. 2002. Archives, Memory, and Interfaces with the Past. Vol. 2.<https:// doi.org/10.1007/BFo2435629> [Consulta: 2 diciembre 2017].

» Hernández Salazar, P. 2011. La importancia de la satisfacción del usuario. En Documentación de las Ciencias de la Información. Vol. 34, 349-368.

»Jaén García, L. F. 2006. La aplicabilidad de los estudios de usuarios en los archivos: el caso de los archivos históricos. En Revista Códice. Vol. 2, no. 1, 45-52.

" Jaén García, L. F. 2013. Los estudios de usuarios en archivos: aplicación en el archivo histórico del archivo nacional de costa rica. En Revista del Archivo Nacional. Vol. 72, no. 1-12, 11-71. 
»Ladrón de Guevara, M. C. 2010. En búsqueda de la satisfacción de los usuarios de archivo. En Anuario Escuela de Archivología. Vol. II, 8-32.

»Lara Navarra, P. y A. Ruiz Rodríguez. 1999. La difusión de información en los archivos: páginas web. En TRIA. Vol. 6, 151-163.

»Martín Moreno, C. 2007. Metodología de investigación en estudios de usuarios. En Revista General de Informacióny Documentación. Vol.17, no. 2,129-149. <http:// revistas.ucm.es/index.php/RGID/article/view/RGIDo707220129A/9407> [Consulta: 2 diciembre 2017].

»Rubio Hernández, A. 2004. Estudios de usuarios en archivos municipales. En Scire: Representación y organización del conocimiento. Vol. 10, no. 1, 217-236.

»Santaella, R. D. 2005. Metodología de estudios de usuarios de información. Estudio de casos en la Administración Pública. En Textos de la CiberSociedad. Vol. 5. <http://dialnet.unirioja.es/servlet/articulo?codigo $=1166822 \&$ info=resumen \&idioma $=$ SPA $>$ [Consulta: 2 diciembre 2017].

"Sanz Casado, E. 1994. Manual de estudios de usuarios. Madrid: Fundación Germán Sánchez Ruipérez.

» Yakel, E. y D. Torres. 2003. Al: archival intelligence and user expertise. En The American Archivist. Vol. 66, no. 1, 51-78.

» Yakel, E. y D. Malkmus. 2016. Contextualizing Archival Literacy. Chicago: SAA. 
